\title{
ANHANG
}

\section{ITAS-Publikationen und Vorträge 2002}

\section{Monografien}

Grunwald, A.: Technikfolgenabschätzung - Eine Einführung. Berlin: edition sigma 2002 (Gesellschaft - Technik - Umwelt, Neue Folge 1)

Halbritter, G.; Bräutigam, K.-R.; Fleischer, T.; Fulda, E.; Georgiewa, D.; Klein-Vielhauer, S.; Kupsch, Chr.: Verkehr in Ballungsräumen. Mögliche Beiträge von Telematiktechniken und-diensten für einen effizienteren und umweltverträglicheren Verkehr. Berlin: Erich Schmidt 2002 (Beiträge zur Umweltgestaltung, Bd. A 149) Hocke, P.: Massenmedien und lokaler Protest. Empirische Fallstudie zur Medienselektivität in einer westdeutschen „Bewegungshochburg“. Wiesbaden: Westdeutscher 2002

Meyer, R.; Börner, J.: Bioenergieträger - eine Chance für die „Dritte Welt“. Verfahren - Realisierung - Wirkungen. Berlin: edition sigma 2002 (Studien des Büros für Technikfolgen-Abschätzung beim Deutschen Bundestag, Bd. 11)

Paschen, H.; Wingert, B.; Coenen, Chr.; Banse, G.: Kultur - Medien - Märkte. Medienentwicklung und kultureller Wandel. Berlin: edition sigma 2002 (Studien des Büros für Technikfolgen-Abschätzung beim Deutschen Bundestag, Bd. 12)

Stehr, N.: Knowledge and Economic Conduct: The Social Foundations of the Modern Economy. Toronto: University of Toronto 2002

\section{Sammelbände}

Banse, G.; Grunwald, A.; Rader, M. (Hrsg.): Innovations for an e-Society. Challenges for Technology Assessment. Berlin: edition sigma 2002 (Gesellschaft - Technik - Umwelt, Neue Folge 2)

Banse, G.; Kiepas, A. (Hrsg.): Rationalität heute - Vorstellungen, Wandlungen, Herausforderungen. Münster: LIT 2002 (Technikphilosophie, Bd. 9)

Banse, G.; Reher, E.-O. (Hrsg.): Allgemeine Technologie - Vergangenheit, Gegenwart, Zukunft. Berlin: Trafo 2002

Brand, K.-W. (Hrsg.): Politik der Nachhaltigkeit. Voraussetzungen, Probleme, Chancen - eine kritische Diskussion. Berlin: edition sigma 2002 (Global zukunftsfähige Entwicklung - Perspektiven für Deutschland, Bd. 3)

Grunwald, A. (Hrsg.): Technikgestaltung für eine nachhaltige Entwicklung. Von der Konzeption zur Umsetzung. Berlin: edition sigma 2002 (Global zukunftsfähige Entwicklung - Perspektiven für Deutschland, Bd. 4)

\section{Aufsätze}

Achternbosch, M.; Bräutigam, K.-R.: Stoffstromanalysen - Einführung in den Schwerpunkt. Technikfolgenabschätzung - Theorie und Praxis, 11(2002)1, S. 7-9

Achternbosch, M.; Bräutigam, K.-R.; Richers, U.: Analyse des Einsatzes von Abfällen als Sekundärbrennstoffe in Zementwerken - Derzeitige Situation, Potentiale und Stoffströme. In: Stein, G. (Hrsg.): Umwelt und Technik im Gleichklang. Technikfolgenforschung und Systemanalyse in Deutschland. Berlin u. a.: Springer 2002, S. $387-404$

Achternbosch, M.; Bräutigam, K.-R.; Vehlow, J.: Transferfaktoren. In: Deutsche Vereinigung für Verbrennungsforschung e.V. (Hrsg.): Thermische Verfahren der Abfallbehandlung - Entwicklungen, Optimierung, Bewertung. Weimar: Universitätsverlag 2002, S. 165-174

Achternbosch, M.; Richers, U.: Comparison of Fabric Filter and Electrostatic Precipitator with Material Flow Balances. In: IT3 Conference (Hrsg.): Twenty-first Annual. International Conference on Incineration and Thermal Treatment Technologies. New Orleans: University of Maryland 2002, p. 1-10

Achternbosch, M.; Richers, U.: Vergleich der abwassererzeugenden und abwasserfreien Rauchgasreinigung von Abfallverbrennungsanlagen. Müll und Abfall, 34(2002)6, S. 333-337 
Arlt, A.: Frithjof Staiß: Jahrbuch Erneuerbare Energien 2001 - Buchvorstellung. Technikfolgenabschätzung Theorie und Praxis, 11(2002)1, S. 120-122

Arlt, A.; Leible, L.; Seifert, H.; Nieke, E.; Fürniß, B.: Processing of Sewage Sludge for Energetic Purposes A Challenge for Process Technology. Bioprocessing of Solid Waste \& Sludge, 2(2002)1, p. 18-28

Banse, G.: Die Zuverlässigkeit zwischen der Tatsächlichkeit und der Fraglichkeit. In: Kiepasa, A. (Hrsg.): Czlowiek wobec wyzwan racjonalnosci. Katowice: Wydawnictwo Uniwersytetu Slaskiego 2002, S. 32-44

Banse, G.: Erfinden im Spannungsfeld von Methodik, Heuristik und Kreativität. In: Bagdazarian, N.G. (Hrsg.): Ethische Herausforderungen der Ingenieurtätigkeit. Dubna: Universitätsverlag 2002, S. 58-64 (Materialien des V. Engelmeyer-Kongresses)

Banse, G.: Günter Ropohl (Hrsg.): Erträge der Interdisziplinären Technikforschung. Eine Bilanz nach 20 Jahren. In: Krebs, H.; Gehrlein, U.; Pfeiffer, J.; Schmidt, J.C. (Hrsg.): Perspektiven Interdisziplinärer Technikforschung - Rezensionen. Technikfolgenabschätzung - Theorie und Praxis, 11(2002)3/4, S. 155-160

Banse, G.: Johann Beckmann und die Folgen. Allgemeine Technologie in Vergangenheit und Gegenwart. In: Banse, G.; Reher, E.-O. (Hrsg.): Allgemeine Technologie - Vergangenheit, Gegenwart, Zukunft. Berlin: Trafo 2002, S. 17-46

Banse, G.: Über den Umgang mit Ungewissheit. In: Banse, G.; Kiepas, A. (Hrsg.): Rationalität heute. Vorstellungen, Wandlungen, Herausforderungen. Münster u. a.: LIT 2002, S. 211-234 (Technikphilosophie, Bd. 9)

Banse, G.; Berg, I. von; Rader, M.: Der Beginn einer neuen Tradition? Der Internationale Kongress ,Innovations for an e-Society. Challenges for Technology Assessment “. TEORIE VEDY, XI(2002)1, S. 121-144

Banse, G.; Reher, E. O.: Symposium „Allgemeine Technologie - Vergangenheit und Gegenwart“ - Tagungsbericht. Technikfolgenabschätzung - Theorie und Praxis, 11(2002)1, S. 141-143

Bechmann, G.; Beck, S.: E-Government: Chancen zur Rationalisierung und Demokratisierung der Verwaltung? Technikfolgenabschätzung - Theorie und Praxis, 11(2002)3/4, S. 5-13

Bechmann, G.; Grunwald, A.: Experimentelle Politik und die Rolle der Wissenschaften in der Umsetzung von Nachhaltigkeit. In: Brand, K.-W. (Hrsg.): Politik der Nachhaltigkeit Voraussetzungen, Probleme, Chancen - eine kritische Diskussion. Berlin: edition sigma 2002, S. 113-130 (Global zukunftsfähige Entwicklung - Perspektiven für Deutschland, Bd. 3)

Bechmann, G.; Stehr, N.: The Legacy of Niklas Luhmann. Society, 39(2002)2, p. 67-75

Bechmann, M.; Werner, M.: Digitales Rathaus zwischen Angebot und Bürgernutzung. Technikfolgenabschätzung - Theorie und Praxis, 11(2002)3/4, S. 68-81

Böhle, K.: Defining the European Commission's Role in the ERA of Foresight-Tagungsbericht. Technikfolgenabschätzung - Theorie und Praxis, 11(2002)2, S. 136-141

Böhle, K.: Integration von Payment Systemen - Bindung mit Hindernissen ECIN, 21.02.2002 (http://www.ecin.de/zahlungssysteme/integration/)

Böhle, K.: Internet-Zahlungssysteme in der Europäischen Union. In: Ketterer, K.-H.; Stroborn, K. (Hrsg.): Handbuch ePayment. Köln: Deutscher Wirtschaftsdienst 2002, S. 45-61

Böhle, K.: The Innovation Dynamics of Internet Payment Systems Development. The IPTS Report, (2002)63, p. 26-33

Böhle, K.: The electronic Payment Systems Observatory - ePSO. Technikfolgenabschätzung - Theorie und Praxis, 11(2002)2, S. 114-125

Böhle, K.; Krueger, M.: Payment Cultures and Internet Payment Systems - Comparing EU and US. Wirtschaftspolitische Blätter, 49(2002)2, p.137-145

Coenen, R.: Sustainable Development - New Challenges for Technology Assessment. In: Jamison, A.; Rohracher, H. (Hrsg.): Technology Studies \& Sustainable Development. München u. a.: Profil 2002, S. 127-143 (Technology and Science Research, Volume 39)

Coenen, R.: Umlenken auf nachhaltige Technologiepfade. In: Grunwald, A. (Hrsg.): Technikgestaltung für eine nachhaltige Entwicklung. Von der Konzeption zur Umsetzung. Berlin: edition sigma 2002, S. 389-405 (Global zukunftsfähige Entwicklung - Perspektiven für Deutschland, Bd. 4)

Ertunc, T.; Hartlieb, N.; Berns, A.; Klein, W.; Schaeffer, A.: Investigations on the binding mechanism of the herbicide simazine to dissolved organic matter in leachates of compost. Chemosphere, 49(2002)6, S. 597-604

Fleischer, T.: Rolf Wüstenhagen: Ökostrom - Von der Nische zum Massenmarkt. Entwicklungsperspektiven und Marketingstrategien für eine zukunftsfähige Elektrizitätsbranche - Rezension. Technikfolgenabschätzung - Theorie und Praxis, 11(2002)1, S. 122-123

Fleischer, T.: Technikfolgenabschätzungen zur Nanotechnologie-Inhaltliche und konzeptionelle Überlegungen. Technikfolgenabschätzung - Theorie und Praxis, 11(2002)3/4, S. 111-122

Fleischer, T.; Grunwald, A.: Technikgestaltung für mehr Nachhaltigkeit - Anforderungen an die Technikfolgenabschätzung. In: Grunwald, A. (Hrsg.): Technikgestaltung für eine nachhaltige Entwicklung. Von der Kon 
zeption zur Umsetzung. Berlin: edition sigma 2002, S. 95-146 (Global zukunftsfähige Entwicklung - Perspektiven für Deutschland, Bd. 4)

Fleischer, T.; Halbritter, G.: Vom Service zum Instrument. Auf der Suche nach einer Rolle für die Verkehrstelematik. Internationales Verkehrswesen, 54(2002)3, S. 102-103

Gloede, F.; Hennen, L.: A Difference that makes a Difference? In: Joss, S.; Belucci, S. (Hrsg.): Participatory Technology Assessment. European Perspectives. Westminster: University of Westminster 2002, p. 92-107

Grunwald, A.: Das Technische und das Nicht-Technische. Eine grundlegende Unterscheidung und ihre kulturelle Bedeutung. Karlsruhe: Forschungszentrum Karlsruhe 2002, S. 37-48 (Wissenschaftliche Berichte, FZKA 6697)

Grunwald, A.: Die Realisierung eines Nachhaltigen Konsums - Aufgabe der Konsumenten? In: Scherborn, G.; Weber, Chr. (Hrsg.): Nachhaltiger Konsum - Auf dem Weg zur gesellschaftlichen Verankerung. München: ökom 2002, S. 433-442

Grunwald, A.: Divergente Wahrnehmungen: Technikfolgenabschätzung zwischen Innovationsförderung und Technikkritik. Development and Perspectives, (2002)1, S. 67-82

Grunwald, A.: Ethische Aspekte der Folgenforschung. In: Sommermann, K.-P. (Hrsg.): Folgen von Folgenforschung. Speyer: Forschungsinstitut für öffentliche Verwaltung 2002, S. 17-38 (Speyerer Forschungsberichte 225)

Grunwald, A.: Formale Legitimisierung und Beweisvernunftmäßigkeit bei Technikbildung. In: Kiepas, A. (Hrsg.): Czlowiek wobec wyzwan racjonalnosci. Katowice: Wydawnictwo Uniwersytetu Slaskiego 2002, S. 32-44

Grunwald, A.: Nachhaltigkeit und integrative Modellierung. In: Gethmann, C. F.; Lingner, S. (Hrsg.): Integrative Modellierung zum Globalen Wandel. Berlin u. a.: Springer 2001, S. 71-106 (Wissenschaftsethik und Technikfolgenbeurteilung, Bd. 17)

Grunwald, A.: Nachhaltigkeitsforschung in der Helmholtz-Gemeinschaft. Verknüpfung von konzeptioneller Arbeit, empirischer Forschung und Technologieentwicklung. GAIA, 11(2002)1, S. 31-33

Grunwald, A.: Nachhaltigkeitsforschung in der Helmholtz-Gemeinschaft. Auf dem Weg zum Programm „Nachhaltigkeit und Technik“. Technikfolgenabschätzung - Theorie und Praxis, 11(2002)2, S. 86-89

Grunwald, A.: Normativ, aber nicht normativistisch. Bemerkungen zur Grenze zwischen Wissen, Werten und Bekehrungen als Antwort auf Christian Berg. Technikfolgenabschätzung - Theorie und Praxis, 11(2002)2, S. 155-157

Grunwald, A.: Perspektiven der Nachhaltigkeitsforschung in der Helmholtz-Gemeinschaft. In: Grunwald, A. (Hrsg.): Technikgestaltung für eine nachhaltige Entwicklung. Von der Konzeption zur Umsetzung. Berlin: edition sigma 2002, S. 407-414 (Global zukunftsfähige Entwicklung - Perspektiven für Deutschland, Bd. 4)

Grunwald, A.: Philosophie zur Reflexion von Wissenschaft und Technik - ein Workshop von Universität Marburg und ITAS - Tagungsbericht. Technikfolgenabschätzung - Theorie und Praxis, 11(2002)1, S. 126-129

Grunwald, A.: Rationalität in der gesellschaftlichen Gestaltung oder blinde Evolution ? In: Banse, G.; Kiepas, A. (Hrsg.): Rationalität heute Vorstellungen, Wandlungen, Herausforderungen. Münster u. a.: LIT 2002, S. 191209 (Technikphilosophie, Bd. 9)

Grunwald, A.: Technikeinführung als gesellschaftlicher Lernprozeß. Zur Rolle von Politik und Technikfolgenabschätzung. In: Kloepfer, M. (Hrsg.): Technikumsteuerung als Rechtsproblem. Rechtsfragen der Einführung der Gentechnik und des Ausstiegs aus der Atomenergie. Berlin: Duncker \& Humblot 2002, S. 43-65 (Schriften zum Technikrecht, Bd. 5)

Grunwald, A.: Technikfolgenabschätzung als wissenschaftlicher Beitrag zu gesellschaftlichen Lernprozessen über Technik. In: Stein, G. (Hrsg.): Umwelt und Technik im Gleichklang. Technikfolgenforschung und Systemanalyse in Deutschland. Berlin u. a.: Springer 2002, S. 3-17

Grunwald, A.: Technology Assessment for Shaping the e-Society. In: Banse, G.; Grunwald, A.; Rader, M. (Hrsg.): Innovations for an e-Society. Challenges for Technology Assessment. Berlin: edition sigma 2002, p. 27-42 (Gesellschaft - Technik - Umwelt, Neue Folge 2)

Grunwald, A.: Technology Assessment und Wirtschaft - neue Erkenntnisse? Technikfolgenabschätzung - Theorie und Praxis, 11(2002)3/4, S. 178-181

Grunwald, A.: Wenn Roboter planen: Implikationen und Probleme einer Begriffszuschreibung. In: Rammert, W.; Schulz-Schaeffer, I. (Hrsg.): Können Maschinen handeln? Soziologische Beiträge zum Verhältnis von Mensch und Technik. Frankfurt/Main: Campus 2002, S. 141-160

Grünwald, R.: Verkehr und Nachhaltigkeit - schwierig aber möglich-Maßnahmen für eine nachhaltige Energieversorgung im Bereich Mobilität. TAB-Brief Nr. 23, 2002, S. 30-31

Grünwald, R.; Oertel, D.: Monitoring „Kernfusion“. Technikfolgenabschätzung - Theorie und Praxis, 11(2002)3/4, S. 123-127

Halbritter, G.; Fleischer, T.: Mögliche Beiträge von Verkehrstelematik-Techniken und -Diensten zur Erreichung einer ,nachhaltigen Entwicklung“. In: Stein, G. (Hrsg.): Umwelt und Technik im Gleichklang. Technikfolgenforschung und Systemanalyse in Deutschland. Berlin u. a.: Springer 2002, S. 263-280 
Halbritter, G.; Fleischer, T.: Nachhaltige Entwicklung im Verkehr. Mobilität im Spannungsfeld neuer Techniken und Dienste und wachsender Ansprüche der Gesellschaft. In: Grunwald, A. (Hrsg.): Technikgestaltung für eine nachhaltige Entwicklung. Von der Konzeption zur Umsetzung. Berlin: edition sigma 2002, S. 179-207 (Global zukunftsfähige Entwicklung - Perspektiven für Deutschland, Bd. 4)

Hennen, L.: Umfrage zur Technikakzeptanz - Technikfolgenakzeptanz und Kontroversen über Technik. TABBrief Nr. 23, 2002, S. 24-27

Hocke, P.: Das Auswahlverhalten von Massenmedien bei der Berichterstattung über lokalen Protest. Eine Prüfung der Nachrichtenwert-Theorie anhand medienunabhängiger Demonstrationsdaten. In: Schatz, H.; Nieland, J-U; Rössler, P. (Hrsg.): Politische Akteure in der Mediendemokratie. Opladen u. a.: Westdeutscher 2002

Jörissen, J.: Konzeption für ein Stoffstromrecht. Technikfolgenabschätzung - Theorie und Praxis, 11(2002)1, S. 64-72

Karger, C.; Rösch, Ch.; Fugger, W.-D.: Biotechnologie in der Landwirtschaft. In: Grunwald, A. (Hrsg.): Technikgestaltung für eine nachhaltige Entwicklung. Von der Konzeption zur Umsetzung. Berlin: edition sigma 2002, S. 363-373 (Global zukunftsfähige Entwicklung - Perspektiven für Deutschland, Bd. 4)

Klann, U.; Schulz, V.: Großflächige Ökobilanzen - Anwendungen der umweltbezogenen Input-Output-Analyse. In: Stein, G. (Hrsg.): Umwelt und Technik im Gleichklang. Technikfolgenforschung und Systemanalyse in Deutschland. Berlin u. a.: Springer 2002, S. 49-60

Klein-Vielhauer, S.: Ein Baustein für nachhaltige(re)s Reisen - Tagungsbericht. Technikfolgenabschätzung Theorie und Praxis, 11(2002)1, S. 129-132

Klein-Vielhauer, S.: Netzwerk Stadtlogistik: „Stadtlogistik: Vom Nischenmarkt zur urbanen Komplettlösung“Tagungsbericht. Technikfolgenabschätzung - Theorie und Praxis, 11(2002)1, S. 136

Klein-Vielhauer, S.: Neue Konzepte für den Güterverkehr in Ballungsräumen - Bemühungen in Praxis und Wissenschaft: Eine Betrachtung aus der Sicht von Systemanalyse und Technikfolgenabschätzung. In: Bundesvereinigung Logistik BVL (Hrsg.): Wissenschaftssymposium Logistik der BVL 2002. München: hussverlag 2002, S. 589-609

Klein-Vielhauer, S.: Wissenschaftssymposium Logistik - Tagungsbericht. Technikfolgenabschätzung - Theorie und Praxis, 11(2002)3/4, S. 168-172

Krings, B.-J.: Genderforschung und Technikentwicklung - Einführung in den Schwerpunkt. Technikfolgenabschätzung - Theorie und Praxis, 11(2002)2, S. 5-8

Krings, B.-J.: Homo Technicus - Wissenschafts- und Technikentwicklung aus Sicht der Feministischen Theorie. Technikfolgenabschätzung - Theorie und Praxis, 11(2002)2, S. 9-20

Krings, B.-J.; Moniz, A.: Competencia o cooperación. Sobre el desarrollo sociocultural de la sociedad de la información. Sociologiá del Trabajo, (2002)44, S. 125-143

Leible, L.; Arlt, A.; Seifert, H.; Kälber, S.; Nieke, E.; Wintzer, D.; Fürniß, B.: Energie aus biogenen Abfällen - Stand und Perspektiven in Deutschland. In: Deutsche Wissenschaftliche Gesellschaft für Erdöl, Erdgas und Kohle e.V. (Hrsg.): Tagungsbericht 2002-2. Velen/Westf.

Leible, L.; Arlt, A.; Seifert, H.; Kälber, S.; Nieke, E.; Fürniß, B.: Energie aus biogenen Reststoffen und Abfällen. In: Grunwald, A. (Hrsg.): Technikgestaltung für eine nachhaltige Entwicklung. Von der Konzeption zur Umsetzung. Berlin: edition sigma 2002, S. 279-298 (Global zukunftsfähige Entwicklung - Perspektiven für Deutschland, Bd. 4)

Meyer, R.: Nahrungsmittel - Entwicklungstendenzen: Ein Ausblick. TAB-Brief Nr. 22, 2002, S. 16-17

Meyer, R.: Nahrungsmittel - Qualität: Wie viel darf es sein? TAB-Brief Nr. 22, 2002, S. 7-10

Meyer, R.; Sauter, A.: Nahrungsmittel aus der Region - für die Region? TAB-Brief Nr. 22, 2002, S. 11-13

Meyer, R.; Sauter, A.: Nahrungsmittel: Potenziale bei Qualität und Regionalisierung der Nahrungsmittelversorgung. TAB-Brief Nr. 22, 2002, S. 5-6

Moniz, A. B.; Van Hootegem, G.; Krings, B.-J.: Technological Practices in the European Auto Industry: Exploring Cases from Belgium, Germany and Portugal. International Journal of Automative Technology and Management, 2(2002)1, p. 84-100

Oertel, D.: Die Verpackung macht den Geschmack - Technologische Trends bei Getränkeverpackungen. TABBrief Nr. 23, 2002, S. 32-33

Oertel, D.: Strom aus Erdwärme: Potenziale und Nutzungschancen - Geothermische Stromerzeugung in Deutschland. TAB-Brief Nr. 23, 2002, S. 28-29

Orwat, C.; Petermann, Th.; Riehm, U.: Elektronischer Handel und Nachhaltigkeit. In: Grunwald, A. (Hrsg.): Technikgestaltung für eine nachhaltige Entwicklung. Von der Konzeption zur Umsetzung. Berlin: edition sigma 2002, S. 245-275 (Global zukunftsfähige Entwicklung - Perspektiven für Deutschland, Bd. 4)

Petermann, Th.: Die militärische Nutzung des Weltraums: frostige Zeiten für Rüstungskontrolle - Neue Technologien und Rüstungskontrolle. TAB-Brief Nr. 23, 2002, S. 21-23 
Revermann, Ch.: Wie viel Strahlung verträgt der Mensch? - Gesundheitliche Aspekte der Mobilfunktechnologie. TAB-Brief Nr. 23, 2002, S. 16-20

Reßler, B.; Achternbosch, M.; Bräutigam, K.-R.; Kupsch, Chr.; Sardemann, G.: Stoffstromanalysen zum Einsatz von carbonfaserverstärkten Kunststoffen im Flugzeugbau. Technikfolgenabschätzung - Theorie und Praxis, 11(2002)1, S. 41-50

Riehm, U.: Digitale Güter in der Buch- und Musikbranche - ein lohnendes Feld für die Technikfolgenabschätzung. In: Stein, G. (Hrsg.): Umwelt und Technik im Gleichklang. Technikfolgenforschung und Systemanalyse in Deutschland. Berlin u. a.: Springer 2002, S. 181-198

Riehm, U.: E-Commerce bleibt auf der Agenda von Wirtschaft und Politik. TAB-Brief Nr. 23, 2002, S. 12-15

Riehm, U.; Orwat, C.; Petermann, Th.: Stand, Perspektiven und Folgen des E-Commerce. In: Weinhardt, Ch.; Holtmann, C. (Hrsg.): E-Commerce - Netze, Märkte, Technologien. Heidelberg: Physica 2002, S. 1-18

Rösch, Ch.: Trends in der Ernährung - eine nachhaltige Entwicklung? In: Scherborn, G.; Weber, Chr. (Hrsg.): Nachhaltiger Konsum - Auf dem Weg zur gesellschaftlichen Verankerung. München: ökom 2002, S. 269-278

Rösch, Ch.; Backhaus, R.; Meyer, B. C.: Nachhaltige Landwirtschaft in Betrieb und Landschaft. In: Grunwald, A. (Hrsg.): Technikgestaltung für eine nachhaltige Entwicklung. Von der Konzeption zur Umsetzung. Berlin: edition sigma 2002, S. 209-244 (Global zukunftsfähige Entwicklung - Perspektiven für Deutschland, Bd. 4)

Seht, H. von : Socio-Economic Impacts of local Environmental Policies. An Analysis for the Field of Climate Protection. Local Environment, 7(2002)1, p. 23-34

Stehr, N.: Biotechnology and the Governance of Knowledge. In: Kulturwissenschaftliches Institut (Hrsg.): Jahrbuch 2001/2002. Essen: Kulturwissenschaftliches Institut 2002, p. 304-316

Stehr, N.: Die Macht des Schwachen. In: Rapp, H.; Scheilke, Ch. Th.; Schmidt, H. (Hrsg.): Zukunftsfähige Bildung und Protestantismus. Stuttgart: Calwer 2002, S. 90-100

Stehr, N.: The Social Role of Knowledge. In: Genov, N. (Hrsg.): Advances in Sociological Knowledge. Paris: International Social Science Council 2002, p. 84-113

Stehr, N.: Wissen. In: Engel, Ch.; Halfmann, J.; Schulte, M. (Hrsg.): Wissen-Nichtwissen-Unsicheres Wissen. Freiburg: Nomos 2002, S. 17-33

Stehr, N.; Storch, H. von : Das Klima in den Köpfen der Menschen. In: Hauser, W. (Hrsg.): Klima. Das Experiment Mit dem Planeten Erde. München: Deutsches Museum 2002, S. 280-291

Stehr, N.; Storch, H. von : Towards a History of Ideas of Anthropogenic Climate Change. In: Wefer, G.; Berger, W. H.; Behre, K.-E.; Jansen, E. (Hrsg.): Climate and history in the North Atlantic Realms. Heidelberg: Springer 2002, p. 17-23

Stelzer, V.: B. Hillebrand, K. Löbbe, H. Clausen, J. Dehio, M., Halstrick-Schwenk, H.D. von Loeffelholz, W. Moos, K.-H. Storchmann: Nachhaltige Entwicklung in Deutschland - Ausgewählte Problemfelder und Lösungsansätze Rezension. Technikfolgenabschätzung - Theorie und Praxis, 11(2002)1, S. 115-117

Stelzer, V.: NRW im Dickicht der Nachhaltigkeits-Indikatoren. Wie misst man Nachhaltigkeit? - Tagungsbericht. Technikfolgenabschätzung - Theorie und Praxis, 11(2002)1, S. 146-147

Stolle, M.: Beeinflusst das Interesse für Technik die Einstellung zur Entsorgung radioaktiver Abfälle? Technikfolgenabschätzung - Theorie und Praxis, 11(2002)3/4, S. 140-149

Weber, A.: Elektronisches Bargeld für mobile Handhabung - Erfahrungen in Asien und Schlussfolgerungen. In: Ketterer, K.-H.; Stroborn, K. (Hrsg.): Handbuch ePayment. Köln: Deutscher Wirtschaftsdienst 2002, S. 62-75

Weber, A.: Enabling Crypto: How Radical Innovations Occur. Communications of the ACM, 45(2002)4, p. 103-107

Wingert, B.: Handhabung von Text - Ergebnisse aus einem Leseselbstversuch mit eBooks. In: Meer, E. van der; Hagendorf, H.; Beyer, R.; Krüger, F.; Nuthmann, A.; Schulz, S. (Hrsg.): 43. Kongress der Deutschen Gesellschaft für Psychologie, Programm - Abstracts. Lengerich u. a.: Pabst Science Publishers 2002, S. 93

Wingert, B.; Skrabe, K.: Design und Psychologie von eBooks - Moderatorenpapier für die AG 2 der 22. MMK 2002 in Münster

\section{Nicht publizierte Vorträge}

Achternbosch, M.; Bräutigam, K.-R.; Kupsch, Chr.; Reßler, B.; Sardemann, G.: Material Flow Analysis - A Comparison of Manufacturing, Use and Fate of CFRP - Fuselage Components versus Aluminium-Components for commercial Airliners. Vortrag beim Workshop „Schwarzer Rumpf“ CFRP for Future Aircraft Fuselage Structures, Braunschweig, 24. Oktober 2002

Arlt, A.; Seifert, H.; Leible, L.; Nieke, E.; Wintzer, D.; Fürniß, B.: Pre-Treatment and Logistics - The Hurdle to turn Biowaste into Biofuels. Lecture: World Renewable Energy Congress VII, Köln, June 29 - July 05, 2002 
Banse, G.: Aktuelle Fragen der Wissenschafts- und Technikethik. Vortrag an der Geisteswissenschaftlichen Fakultät der Matej Bel-Universität, Banska Bystrica, Slowakische Republik, 28. September 2002

Banse, G.: Chancen und Gefahren der Informationstechnik. Vortrag auf der Engelmeyer-Konferenz zum Thema „e-Society“ der Technischen Baumann-Hochschule, Moskau, Russland, 29. März 2002

Banse, G.: Data Flow in Europe - Free and Safe? The Case of Digital Signature. Vortrag auf dem gemeinsamen Symposium „Shaping better Technologies“ der BTU Cottbus und der BUTE Budapest, Budapest, Hungary, 13.-14. April 2002

Banse, G.: Ethische Probleme in der Informationsgesellschaft. Vortrag an der Geisteswissenschaftlichen Fakultät der Matej Bel-Universität, Bystrica, Slowakische Republik, 28. Februar 2002

Banse, G.: Integrative nachhaltige Entwicklung - Nachhaltigkeitsstrategien aus der Sicht der Technikfolgenabschätzung. Vortrag auf der Konferenz der Rosa-Luxemburg-Stiftung Gesellschaftsanalyse und Politische Bildung e.V. „Nachhaltigkeit und soziale Gerechtigkeit im 21. Jahrhundert“, Berlin, 15.-17. November 2002

Banse, G.: Ist nachhaltige Entwicklung ohne Berücksichtigung der Technik möglich? Vortrag auf der Internationalen Konferenz „Wissenschaftlich-technische Entwicklung in historischer Perspektive“, Russische Akademie der Wissenschaften, Moskau, Russland, 1.-2. September 2002

Banse, G.: Konstruktionshandeln - Geschichte und Gegenwart. Vortrag am Institut für Philosophie der Russischen Akademie der Wissenschaften, Moskau, Russland, 26. März 2002

Banse, G.: Neue Medien und Kultur. Vortrag an der Hochschule für Management und Sozialwissenschaften, Tychy, Polen, 9. Oktober 2002

Banse, G.: Sicherheit zwischen Faktizität und Hypothetizität. Vortrag im Institut für Philosophie der Schlesischen Universität, Katowice, Polen, 7. Oktober 2002

Banse, G.: Technikfolgenabschätzung - Neue Medien und Kultur. Vortrag im Institut für Philosophie der Universität Salzburg, Salzburg, Österreich, 15. Mai 2002

Banse, G.: Technikgestaltung im Spannungsfeld von Plan und Lebenswelt. Vortrag auf der Tagung „Technikgestaltung zwischen Wunsch und Wirklichkeit", Technische Universität Darmstadt, 24. Oktober 2002

Banse, G.: Was hat Technik mit Toleranz zu tun? Vortrag zur Gemeinsamen Wissenschaftlichen Konferenz der Leibniz-Sozietät e.V. und des Mittelstandsverbandes Oberhavel e.V. „Toleranz. Ihre historische Genese, ihre Chancen und Grenzen im 21. Jahrhundert", Oranienburg b. Berlin, 26. Oktober 2002

Banse, G.: Zur Wissenschaftstheorie der Technikwissenschaften. Vortrag auf dem XIII. Workshop der Deutschen Gesellschaft für Systemforschung e.V. (DGSF), „Technik - System - Verantwortung“, BTU Cottbus, Cottbus, 11. Juli 2002

Bechmann, G.: Evolution oder Gestaltung? Ist Technik gesellschaftlich gestaltbar? Vortrag auf der Tagung „Technikgestaltung zwischen Wunsch und Wirklichkeit“, Technische Universität Darmstadt, 25. Oktober 2002

Bechmann, G.: Nachhaltigkeit in der Informationsgesellschaft. Vortag an der Internationalen Unabhängigen Universität für Ökologie und Politologie (MHEPU), Moskau, Russland, 20. September 2002

Bechmann, G.: Neue Formen wissenschaftlicher Produktion. Vortrag bei der Russischen Akademie der Wissenschaft, Moskau, Russland, 19. September 2002

Bechmann, G.: The Globalization of Communication - The Internet as a new Form of Computer-mediated Communication. Lecture: Society for Phemenology, 4th Annual Conference, Pueblo, Mexico, February 20-24, 2002

Bechmann, G.: Wissenschaftsproduktion im Übergang von der Industriegesellschaft zur Wissensgesellschaft. Vortrag am Institut für Wissenschafts- und Technikforschung, Universität Bielefeld, 16. Dezember 2002

Bechmann, G.: Zur Funktion der Ethik im Globalisierungsprozess. Vortrag beim Dritten Russischen Philosophie-Kongress, Rostow am Don, Russland, 17. September 2002

Bräutigam, K.-R.: Einsatz von Sekundärbrennstoffen und Sekundärrohstoffen zur Herstellung von Klinker und Zement. Vortrag beim Seminar Abfallwirtschaft, Institut für Wassergüte und Abfallwirtschaft, Technische Universität Wien, Wien, Österreich, 4. März 2002

Bräutigam, K.-R.; Achternbosch, M.; Hartlieb, N.: Co-Incineration of Wastes in Cement Kilns - A Comparison of calculated and measured Concentration Values of Heavy Metals in Cement. Lecture: IT3'02 Conference, New Orleans, Louisiana, May 13-17, 2002

Coenen, R.: Research and Development Policy in Support of Sustainable Development. Lecture: CIPRE's Second Mid-career Seminar, Budapest, Hungary, November 17-26, 2002

Coenen, R.: Technology Assessment in Europe. Lecture: CIPRE's Second Mid-career Seminar, Budapest, Hungary, November 17-26, 2002

Fleischer, T.; Oertel, D.: Brennstoffzellen-Technologie - Ausgewählte Ergebnisse einer Technology-AssessmentStudie für den Deutschen Bundestag. Vortrag auf der Sitzung des VDI-GVC-Fachausschusses „Energieverfahrenstechnik“, Würzburg, 13.-14. März 2002 
Grunwald, A.: Das Bild vom Menschen in den Wissenschaften. Zwischen homo faber und Untersuchungsobjekt. Vortrag anlässlich des Treffens des Erzbischofs mit den Hochschullehrern, Freiburg, 29. April 2002

Grunwald, A.: Das Schreckgespenst des technologischen Determinismus und seine Überwindung. Vortrag auf der Tagung „Technikgestaltung zwischen Wunsch und Wirklichkeit“, Technische Universität Darmstadt, 24. Oktober 2002

Grunwald, A.: Die Grenzen der Technik in Natur und Kultur - Sinn und Unsinn einer Fragestellung. Vortrag beim Deutschen Kongress für Philosophie, Bonn, 26. September 2002

Grunwald, A.: Die anthropologische Bedeutung des Technischen. Konsequenzen für nachhaltige Technikgestaltung. Vortrag beim Osteuropa-Kolleg an der Universität Karlsruhe, Karlsruhe, 7. Mai 2002

Grunwald, A.: Gesellschaftliche Technikgestaltung und die Herausforderungen an interdisziplinäre Technikforschung. Vortrag am Institut für Philosophie der Russischen Akademie der Wissenschaften, Moskau, Russland, 23. Mai 2002

Grunwald, A.: Neue Formen der Wissensproduktion. Vortrag: DFG Rundgespräch „Das Unerforschte“. Konferenz über Nichtwissen und Forschungslücken in Bauwesen und Planungswissenschaften, Dortmund, 5. Dezember 2002

Grunwald, A.: Partizipative Technikfolgenabschätzung - offene Fragen und kritische Bemerkungen. Vortrag beim ARC-Forum zur Systemforschung Technik-Wirtschaft-Umwelt, Seibersdorf, 17. Januar 2002

Grunwald, A.: Partizipative Technikfolgenabschätzung für Zukunftstechnologien: Zwischen Hoffnungen und Illusionen. Vortrag bei der Ringvorlesung, SS 2002, an der TU Darmstadt, Elfenbeinturm oder Stimmzettel? Demokratiefähigkeit von „Zukunftswissenschaften“ und „Zukunftstechnologien“, Darmstadt, 27. Juni 2002

Grunwald, A.: Relevanz und Risiko. Zu Qualitätskriterien integrativer Forschung. Vortrag auf dem Workshop „Normativität und Unsicherheit“, Universität Stuttgart, 22. Februar 2002

Grunwald, A.: Strategien für eine kohärente Klimapolitik. Vortrag: Präsentation der Klimastudie der Europäischen Akademie, Berlin, 6. Mai 2002

Grunwald, A.: Technik nachhaltig gestalten? Vortrag an der Universität für Ökologie und Politologie, Moskau, Russland, 23. Mai 2002

Grunwald, A.: Technikgestaltung als erweitertes Können. Vortrag auf dem XIII. Workshop der Deutschen Gesellschaft für Systemforschung e.V. (DGSF), „Technik - System - Verantwortung“, BTU Cottbus, Cottbus, 11. Juli 2002

Grunwald, A.: Verantwortung für den wissenschaftlichen Fortschritt? Ethik der Technik - Konzepte und Probleme. Vortrag: Ringvorlesung aus Anlass der Einführung des Ethisch-Philosophischen Grundlagenstudiums, Universität Freiburg, 5. Dezember 2002

Grunwald, A.: Wissenschaft als Problemlösung? Fragen an eine Theorie interdisziplinärer Forschung. Vortrag an der Universität Marburg: Gestaltung und Reflexion - Wissenschaft und Technik in philosophischer Kritik, Marburg, 1. Februar 2002

Grunwald, A.; Sauter, A.: Langzeitmonitoring der Freisetzung von GVP. Gesellschaftliche, politische und wissenschaftliche Dimensionen. Vortrag bei der Konferenz „Kontrollierte Freisetzung gentechnisch veränderter Pflanzen“, Berlin, 13. Juni 2002

Halbritter, G.: Wirkungen von Technikfolgenabschätzungen: Die deutsche und internationale Diskussion zur Verkehrstelematik. Vortrag auf dem Workshop „Das vernetzte Fahrzeug - Technologiefolgenabschätzung Verkehrstechnik“, Bern, Schweiz, 26. Februar 2002

Halbritter, G.: Zur Rolle neuer Techniken und Dienste im Verkehr als Beitrag zu einer ,nachhaltigen Entwicklung“. Vortrag auf der Tagung „Das zweite Jahrhundert des Automobils - Perspektiven der Automobilindustrie und des Automobilismus am Beginn des 21. Jahrhunderts“ im Wissenschaftszentrum Berlin für Sozialforschung, Berlin, 10. Januar 2002

Kopfmüller, J.: Anwendung des integrativen Nachhaltigkeitskonzepts der HGF für den Energiebereich. Vortrag im Rahmen der Tagung „Nachhaltigkeit am Beispiel regenerativer Energiesysteme“, veranstaltet vom Lehrstuhl für Energiesysteme und Energiewirtschaft der Ruhr-Universität Bochum, 2. Mai 2002

Kopfmüller, J.: Die globale Dimension des Leitbilds einer nachhaltigen Entwicklung. Vortrag im Rahmen der Tagung „Nachhaltige Entwicklung und Globaler Wandel. Bestandsaufnahme, Bewertungen und Handlungsbedarf“, Bad Honnef, 6.-7. Juni 2002

Kopfmüller, J.: Die ökonomische Dimension nachhaltiger Entwicklung. Vortrag im Rahmen der Vorlesungsreihe „Nachhaltigkeit im Bauwesen“ des Instituts für Industrielle Bauproduktion (ifib) an der Universität Karlsruhe, Karlsruhe, 5. November 2002

Kopfmüller, J.: Nachhaltige Entwicklung: ein integratives Konzept. Vortrag im Rahmen der 1. Interdisziplinären Sommerschule für Nachhaltige Chemie, veranstaltet von der TU München und dem Wissenschaftszentrum Weihenstephan, Freising, 23. Juli 2002 
Krings, B.-J.; Coenen, R.: The German Research Policy for Sustainable Development. Lecture: Workshop „Setting Concepts in Motion: Mapping Common Research Actions Supporting Sustainable Development in Europe“, Seville, Spain, May 23-24, 2002

Leible, L.; Arlt, A.; Kälber, S.; Nieke, E.; Wintzer, D.; Fürniß, B.: Organic Waste for Heat and Power Production - Status Quo and Potential in German Energy Supply. Lecture: World Renewable Energy Congress VII, Köln, June 29 - July 05, 2002

Meyer, R.: Entwicklungen und Perspektiven von TA. Vortrag: Expertenanhörung des Ministeriums für Umwelt, Natur und Forsten des Landes Schleswig-Holstein, Kiel, 18. September 2002

Meyer, R.: Identifizierung von zukünftigem politischem Gestaltungsbedarf als Teil von TA-Prozessen. Vortrag auf der Konferenz TA'02: Technikgestaltung im 21. Jahrhundert, Wien, Österreich, 27. Mai 2002

Oertel, D.; Fleischer, T.: Brennstoffzellensysteme - Technische, ökonomische und ökologische Potenziale. Vortrag an der Fachhochschule Köln im Rahmen der Ringvorlesung „Zukunftstechnik Brennstoffzelle“, Köln, 24. Oktober 2002

Reßler, B.; Achternbosch, M.; Bräutigam, K.-R.; Kupsch, Chr.; Sardemann, G.: Stoffstromanalyse von Carbonfasermaterialien für den Einsatz im Flugzeugbau. Vortrag auf der GDCH-Tagung Umweltchemie, Braunschweig, 6.-8. Oktober 2002

Riehm, U.: Die Buchhandelsbranche vor der Herausforderung des Internets - Projektbericht und Thesen. Vortrag auf der öffentlichen Vortragsreihe „Alles Buch 5: Electronic Publishing und Online-Buchhandel: Ein Markt im Umbruch?" der Universität Erlangen-Nürnberg, Buchwissenschaft, Erlangen, 11. Dezember 2002

Riehm, U.: Online-Buchhandel revisited. Vortrag auf dem Workshop „Electronic Commerce - revisited: Forschungsstand und Forschungsperspektiven“ der Akademie für Technikfolgenabschätzung in Baden-Württemberg, Stuttgart, 5. Dezember 2002

Sauter, A.: Das TAB - Technikfolgenabschätzung im Auftrag des Deutschen Bundestages. Vortrag im Rahmen der Veranstaltung: Gentechnik - aber sicher! Expertenanhörung zur Technikfolgen-Abschätzung, Kiel, 18. September 2002

Stehr, N.: A World made of Knowledge. Lecture: International Conference „New Knowledge and New Consciousness in the Era of the Knowledge Society“, Budapest, Hungary, January 31, 2002

Stehr, N.: Das Produktivitätsparadox. Vortrag: Sektion Wissenschaftsforschung der Deutschen Gesellschaft für Soziologie, Universität München, Juni 2002.

Stehr, N.: Die Chancen und Risiken der Globalisierung: Rückentwicklung oder Fortentwicklung?. Vortrag: 26. Duisburger Akzente, Globalisierungswelten, Duisburg, 14. Juni 2002

Stehr, N.: Mitigation vs Adaptation. Lecture: „Climate change: what can be done?”, $663^{\text {rd }}$ Wilton Park Conference, Wiston House, Shoreham, England, May 13-17, 2002

Stehr, N.: Mitigation vs. Adaptation. Vortrag auf der Tagung „Climate Change: what can be done?”, Wilton Park Conference, Steyning, England, May 13-17, 2002

Stehr, N.: Modern Societies as Knowledge Societies. Lecture: Plenary Address, World Congress of Sociology, Brisbane, Australia, July 9, 2002.

Stehr, N.: Moderne Gesellschaften sind zerbrechliche Gesellschaften. Vortrag: Hamburger Institut für Lehrerfortbildung, Hamburg, 21. Januar 2002

Stehr, N.: Produktivitätsparadox. Vortrag auf der Tagung „Wissenschaft in der Wissensgesellschaft“, München, 7.-8. Juni 2002

Stehr, N.: Society and Climate: Extremes, Mitigation and Adaptation. Lecture: Conference: Climate, Society and environmental Impacts, University Linköping, Sweden, April 9-11, 2002

Stehr, N.: Society and Climate: Extremes, Mitigation and Adaptation. Lecture: Workshop „Climate, society and environmental impacts“, Linköpings Universitet, Norköpping, Sweden, March 25, 2002.

Stehr, N.: The Authority of Complexity: Scientific Knowledge and public Policies. Lecture: Department of Sociology, University of Alberta, Edmonton, Alberta, Canada, November 18, 2002.

Stehr, N.: The social Role of Knowledge. Lecture: International Conference on Social Science and Social Policy in the $21^{\text {st }}$ Century (in celebration of the 50th Anniversary of the International Social Science Council, Vienna International Centre, Vienna, Austria, December 9, 2002. 


\section{Forschungsberichte}

Achternbosch, M.; Richers, U.: Material Flows and Investment Costs of Flue Gas Cleaning Systems of Municipal Solid Waste Incinerators. Karlsruhe: Forschungszentrum Karlsruhe 2002 (Wissenschaftliche Berichte, FZKA 6726)

Banse, G.; Meier, B.; Wolffgramm, H. (Hrsg.): Technikbilder und Technikkonzepte im Wandel - eine technikphilosophische und allgemeintechnische Analyse. Karlsruhe: Forschungszentrum Karlsruhe 2002 (Wissenschaftliche Berichte, FZKA 6697)

Böhle, K.: Integration of Electronic Payment Systems into B2C Internet-Commerce - Problems and Perspectives. Seville: European Communities 2002 (Electronic Payment Systems Observatory (ePSO), No. 8, EUR 20277 EN)

Grunwald, A.; Grünwald, R.; Oertel, D.; Paschen, H.: Kernfusion - Sachstandsbericht. Berlin: Büro für Technikfolgen-Abschätzung beim Deutschen Bundestag (TAB) 2002 (TAB-Arbeitsbericht Nr. 75)

Grünwald, R.; Oertel, D.; Paschen, H.: Maßnahmen für eine nachhaltige Energieversorgung im Bereich Mobilität - Sachstandsbericht. Berlin: Büro für Technikfolgen-Abschätzung beim Deutschen Bundestag (TAB) 2002 (TABArbeitsbericht Nr. 79)

Meyer, R.; Sauter, A.: Entwicklungstendenzen von Nahrungsmittelangebot und -nachfrage und ihre FolgenBasisanalysen. Berlin: Büro für Technikfolgen-Abschätzung beim Deutschen Bundestag (TAB) 2002 (TABArbeitsbericht Nr. 81)

Oertel, D.; Petermann, Th.; Scherz, C.: Technologische Trends bei Getränkeverpackungen und ihre Relevanz für Ressourcenschonung und Kreislaufwirtschaft. Berlin: Büro für Technikfolgen-Abschätzung beim Deutschen Bundestag (TAB) 2002 (TAB-Hintergrundpapier Nr. 9)

Orwat, C.: Innovationsbedingungen des E-Commerce - der elektronische Handel mit digitalen Produkten. Berlin: Büro für Technikfolgen-Abschätzung beim Deutschen Bundestag (TAB) 2002 (TAB-Hintergrundpapier Nr. 8)

Petermann, Th.; Sauter, A.: Biometrische Identifikationssysteme - Sachstandsbericht. Berlin: Büro für Technikfolgen-Abschätzung beim Deutschen Bundestag (TAB) 2002 (TAB-Arbeitsbericht Nr. 76)

Revermann, Ch.; Petermann, Th.: Tourismus in Großschutzgebieten - Wechselwirkungen und Kooperationsmöglichkeiten zwischen Naturschutz und regionalem Tourismus - Endbericht. Berlin: Büro für TechnikfolgenAbschätzung beim Deutschen Bundestag (TAB) 2002 (TAB-Arbeitsbericht Nr. 77)

Riehm, U.: Innovationsbedingungen des E-Commerce - die technischen Kommunikationsinfrastrukturen für den elektronischen Handel. Berlin: Büro für Technikfolgen-Abschätzung beim Deutschen Bundestag (TAB) 2002 (TAB-Hintergrundpapier Nr. 7)

Riehm, U.; Petermann, Th.; Orwat, C.; Coenen, Chr.; Revermann, Ch.; Scherz, C.; Wingert, B.: E-Commerce - Endbericht. Berlin: Büro für Technikfolgen-Abschätzung beim Deutschen Bundestag (TAB) 2002 (TABArbeitsbericht Nr. 78)

Sauter, A.; Hennen, L.; Meyer, R.: Neue Herausforderungen für die deutsche TSE-Forschung und ihre Förderung. Berlin: Büro für Technikfolgen-Abschätzung beim Deutschen Bundestag (TAB) 2002 (TABDiskussionspapier Nr. 9) 